\title{
Chinese EFL Learners' Emotion, Engagement and Language Achievement: A Latent Profile Analysis
}

\author{
Enhao Feng \\ College of Foreign Languages, Zhejiang Normal University, Jinhua, Zhejiang, China
}

\begin{abstract}
Learner engagement and achievement emotion play a significant role in language learning. However, related research is scant in the field of second language acquisition (SLA). The present study sets out to narrow the gap. It adopts a person-centered latent profile analysis, aiming at discovering the unobserved sub-groups and patterns of foreign language enjoyment, foreign language classroom anxiety, behavioral engagement, and self-perceived achievement within 300 Chinese EFL learners. The results indicate that three distinct profiles could be identified, namely high-FLE, low-FLE, and medium-FLE, each of which appears to have a unique pattern of emotions, engagement and self-perceived achievement. The medium-FLE profile takes the largest proportion of all participants, while the high-FLE profile has the least population. Foreign language $(F L)$ teachers are recommended to carry out interventions to improve learners' positive experience in learning FL and to better engage learners in activities to improve their achievement.
\end{abstract}

Keywords: Latent profile analysis, Emotion, Engagement, Second language acquisition, Enjoyment, Anxiety.

\section{Introduction}

Under the influence of positive psychology (PP), the past decade has witnessed a 'positive turn' in the field of second language acquisition (SLA) (Oxford, 2015). Foreign language enjoyment (FLE), a construct first proposed by Dewaele \& MacIntyre (2014), has become the most popular positive emotion under investigation. FLE is usually studied together with foreign language classroom anxiety (FLCA), which is almost the most researched emotion in SLA. A review of the literature reveals a moderate correlation between them, suggesting a degree of inter-relationship but essentially independent of the dimensions (Dewaele et al., 2018; Dewaele \& Alfawzan, 2018; Jiang \& Dewaele, 2019). It is argued by Dewaele \& MacIntyre (2016) that positive and negative emotions are complementary, similar to the left and right feet of learners and that learners must regulate both emotions.

Although the correlation between FLE and FLCA is relatively well studied, how the two emotions are related to other learner variables is not equally well-investigated, for example, learner engagement. Seligman (2018) established an influential model of psychological well-being and happiness called 'PERMA', and each of the letters stands for the fundamental building block for well-being. "P" stands for Positive emotion and the ability to remain optimistic, while "E" stands for Engagement with activities that use one's character strengths. Now, positive emotions are gradually acknowledged by SLA researchers, however, engagement is still marginalized. Learner engagement is significant in language learning, especially in terms of actual learning and acquisition. Learners may be motivated, yet they may then become distracted and disengaged in the activities at hand. There can be no learning or progress without engagement, especially in skills-based acquisition (Angelovska \& Mercer, 2021). When compared with the field of education where a large body of research on learner engagement is conducted, engagement study in SLA is still in its infancy, and this is especially true in
China. Therefore, it is urgent to conduct a study on L2 engagement.

The present study aims at discovering the profiles of Chinese EFL learners' FLE, FLCA, engagement, and self-perceived achievement (SPA) through a person-centered latent profile analysis, which has seldom been utilized in SLA. Although many studies have indicated the possible relationships between achievement emotions and engagement (Khajavy, 2021; Guz \& Tetiurka, 2016; Oga-Baldwin, 2019), relevant SLA studies are surprisingly scant, the present study hence hopes to offer insights into the correlations between them in SLA, and provide a fine-grained emotion and engagement profile of Chinese EFL learners, which may shed some light on the teaching of EFL in China.

\section{Literature Review}

\subsection{Foreign Language Classroom Anxiety and Foreign} Language Enjoyment

FLCA was first proposed in Horwitz et al. (1986), in which they elicited feedbacks from FL students regarding anxiety-inducing triggers in their FL classrooms, such as the worry of not performing well on tests, speaking out in front of others, and possibly unfavorable reactions from classmates and professors. Together, these sources of FL anxiety coalesce into a "conceptually distinct variable in FL learning" (p. 125), namely FLCA. It is "a distinct complex of self-perceptions, beliefs, feelings, and behaviors related to classroom learning arising from the uniqueness of the language learning process" (Horwitz et al., 1986 p. 128). Anxiety is multifaceted (Horwitz, 2010, p.145). According to Spielberger and Reheiser (2009), there are two types of anxiety: state anxiety and trait anxiety, with the former referring to "the intense subjective feelings of tension, apprehension, fear, nervousness, and worry in response to a specific situation at a specific time, with its associated arousal of the automatic nervous system (p.276)." Trait anxiety, on the other hand, is a personality trait, and persons with high 
trait anxiety are more likely to feel nervous in certain situations than those with low trait anxiety (Alpert \& Haber, 1960). That is, high trait anxiety, like any other stable quality, predisposes a person to anxiety in a range of situations. FLCA is a type of state anxiety that occurs in certain classroom conditions (Horwitz et al., 1986). FLCA has been repeatedly found to be negatively correlated with language performance since Horwitz et al. (1986)'s influential research (Horwitz, 2000; Teimouri, Goetze, \& Plonsky, 2019).

With the emergence of PP in SLA, the other side of the same coin, namely positive emotion, is now drawing increasing research attention. Researchers of PP want to know how language learners may develop and maintain their motivation, perseverance, and resiliency, as well as positive emotions necessary for the long-term undertaking of learning a foreign language' (MacIntyre \& Mercer, 2014, p. 156). Positive emotions are thought to have positive consequences on language acquisition, such as increased attention, focus in class, resilience in coping with problems, and the development of personal resources (Fredrickson, 2001; MacIntyre \& Gregersen, 2012).

Dewaele, MacIntyre, and colleagues (Dewaele et al., 2019) conducted a series of groundbreaking research that has been leading in applying PP to SLA, with a particular focus on FLE. In their seminal work, Dewaele \& MacIntyre (2014) collected data from 1,742 participants all around the world, representing 90 different nationalities, and found the construct of FLE. In the same study, they invented the FLE scale (FLES), which has 21 items in total. Later the same author compacted the 21-item FLES into a 14-item one, with two inner structures being identified, namely FLE-Social and FLE-Private (Dewaele \& MacIntyre, 2016). More recently, a series of studies further compacted the FLES into a 10-item one (Dewaele \& Dewaele, 2017; Dewaele et al., 2017; Saito et al., 2018). In China, the study of positive emotion is just beginning. Li et al. (2018) collected data from 1,718 Chinese participants and refined the 16-item FLES (Dewaele \& MacIntyre, 2016) into an 11-item Chinese Version of Foreign Language Enjoyment Scale (CFLES) whose psychometric properties were then examined. The CFLES was adopted later in many studies in China (e.g., Li et al., 2020; Li, 2020).

Under the influence of the second-wave positive psychology or "PP 2.0" in SLA (MacIntyre, 2021), researchers are picking up a more holistic view of emotions, as argued by MacIntyre (2021), "is both more social and more integrative than the original concept of PP, in the sense that it doesn't emphasize positivity to the exclusion of negativity, rather it emphasizes how positivity and negativity work together in social contexts." The growing body of research on the correlations between FLE and FLCA is evidence. A brief review of the literature reveals that FL learners generally experienced more FLE than FLCA in their classrooms, a pattern that was first found in Dewaele \& MacIntyre (2014) and confirmed later in numerous studies (Botes, 2021; Dewaele \& MacIntyre, 2019; Dewaele, Witney, Saito \& Dewaele, 2018; Jiang \& Dewaele, 2019). However, as acknowledged by Dewaele (2021), since data was acquired using snowball or convenience sampling, this conclusion cannot be applied to the whole learner population. As a result, it is likely that self-selected participants were more likely to be good and motivated students. The relationship between FLE and FLCA is now well studied, they are like Janus' two faces (Dewaele \& MacIntyre, 2014). Dewaele and MacIntyre (2014) discovered a modest negative correlation $(\mathrm{r}=-.36)$ between FLE and FLCA, indicating that the dimensions have some interrelationship but are largely independent. In the qualitative part, participants' accounts of fun moments in the FL classroom double-checked this conclusion. The independence of FLE and FLCA was also validated in Dewaele and MacIntyre (2016). The authors stated that these positive and negative emotions are complementary, similar to the left and right foot of learners and that learners must control both feelings. A meta-analysis reveals a moderate negative correlation between FLE and FLCA ( $r=-.29, k=17, N=9514)$, suggesting that learners who experience more FLE typically suffer less from FLCA (Dewaele, Botes \& Greiff, 2021).

Although FLCA is generally reported to be negatively and moderately related to FLE, a high level of FLE and FLCA sometimes can appear simultaneously in the same context. Quantitative studies have found a prevailing moderate negative correlation between FLCA and FLE (Botes et al., 2020a; Dewaele \& MacIntyre, 2014, 2016; Khajavy, MacIntyre, \& Barabadi, 2018), yet mixed-method studies are rife with reports of students experiencing high levels of FLCA and FLE simultaneously (Dewaele, MacIntyre, Boudreau, \& Dewaele, 2016; Dewaele, Özdemir, et al., 2019). Numerous mixed-methods research studies have presented cases of language learners displaying high levels of language anxiety in conjunction with high levels of proficiency/achievement (Dewaele \& Alfawzan, 2018; Gkonou, 2017; MacIntyre, 1995) despite the prevailing moderate negative correlation between language anxiety and academic achievement. Furthermore, FL learners have also displayed low levels of enjoyment in combination with high levels of perceived proficiency (Li et al., 2019). Therefore, many combinations are possible with regard to FL learners' emotions, with learners displaying equally high, equally low, or opposing levels of FLCA and FLE. To probe into the possible patterns of FLE and FLCA, the traditional variable-centered approach is insufficient, therefore, the present study adopted a person-centered approach.

\subsection{Learner Engagement}

Engagement is one of the hottest topics in educational psychology and has even been described as 'the holy grail of learning' (Sinatra et al. 2015: p.1). The importance of engagement has long been recognized, and it has been related to increased achievement, motivation, and interest, all of which are desirable characteristics in the educational process (Christenson, Reschly, \& Wylie, 2012). However, engagement has not received sufficient attention in SLA yet. This may partly be due to the novelty of the construct, which was said to be a "new kid on the block" compared with popular construct as motivation (Reschly and Christenson 2012, p.14). Given its emphasis on active behaviors, engagement is particularly important and appealing in language learning (Mercer, 2019). Since learners who are motivated may become distracted and not engaged in the learning activities, while those who are engaged can truly get involved in the learning process, hence engagement usually indicates "real" learning and acquisition (Angelovska \& 
Mercer, 2021). So, engagement deserves more attention in SLA.

Engagement is a multi-dimensional concept with three fundamental facets: behavioral, emotional, and cognitive aspects (Fredricks et al., 2004). Later, Svalberg (2009) introduced a social component, and more recently, agentic engagement was offered as a new dimension (Reeve \& Tseng, 2011). Behavioral engagement is reflected by the amount and quality of learners' active participation in learning, and early L2 research operationalized behavioral engagement by gauging word counts and turn counts (Bygate \& Samuda, 2009; Dörnyei \& Kormos, 2000; Platt \& Brooks, 2002). Learners' behavioral engagement in L2 learning, for example, involves their spontaneous participation in speaking, interactional initiative, duration on task, the quantity of semantic material generated while on task, and perseverance on task without the need for help or guidance (Philp \& Duchesne, 2016).

The fundamental facet of engagement, according to Skinner et al. (2008, p.778), is behavioral engagement, whereas cognitive and agentic engagement might be regarded as a subcomponent of behavioral engagement (Skinner, Kindermann, \& Furrer, 2009). Oga-Baldwin (2019) claimed that engagement in class at least begins with behavior. Although still very limited, L2 research suggests that active classroom engagement may lead to students paying greater attention to language forms and meanings, reflecting on their L2 usage, and effectively completing learning activities, resulting in improved performance (Xue et al., 2020).

Besides, engagement is also characterized by several other features, among which the notion of action stands at the center (Skinner \& Pitzer, 2012) because engagement has to do with how actively one is involved in a learning task, it is easy to infer the significance of action; moreover, the behavioral dimension is also the key to distinguishing engagement from motivation (Mercer, 2019), which is one of the reasons for the present study to choose behavioral engagement as the research objective (Skinner \& Pitzer, 2012). The second distinguishing feature is that engagement is context-sensitive (Hiver et al., 2021a). Cultures, communities, families, classrooms, and activities within classrooms all play a role in learners' engagement (e.g., Finn \& Zimmer, 2012; Pianta et al., 2012; Shernoff, 2013). Third, engagement requires a target (Hiver et al., 2021a); it is self-evident that we must first have an objective before being interested in that topic, circumstance, person, or work. Fourth, engagement is both dynamic and changeable (Appleton et al., 2008). Learner engagement may fluctuate and alter as intrapersonal and contextual variables change (Fredricks et al., 2004). Last but not least, engagement is domain-specific (Wang et al., 2016), indicating L2 engagement must be investigated separately and the scale measuring it must also be specialized. Taken together, the abovementioned factors suggest that it is necessary to investigate behavioral L2 engagement in the Chinese EFL context independently.

\subsection{Studies on the Links between FLE, FLCA, and Engagement in SLA}

According to the model of 'PERMA' (Seligman, 2018), both positive emotion and engagement are building bricks of well-being. However, surprisingly, scant attention has been paid to the associations between them in SLA. Although the number of related research is rather limited, still there are a handful of studies on the correlations between FLE, FLCA, and engagement.

Dewaele and Li (2021) investigated the relationship between perceived teacher enthusiasm, student emotions (enjoyment and boredom), and social-behavioral engagement. A total of 2,002 EFL students from 11 Chinese universities took part in the study. The findings indicated a complicated interplay between the three variables. Teachers' enthusiasm influenced social-behavioral engagement both directly and indirectly, according to mediation analysis. The indirect relationship was established through enjoyment and boredom. Both positive and negative emotions, such as enjoyment and boredom, were associated with teacher enthusiasm, and these emotions mediated the influence of students' perceived teacher enthusiasm on their own engagement.

Khajavy (2021) looked into the relationship between FLE, FLCA, grit, engagement, and L2 reading achievement. According to the findings, FLE positively predicted L2 engagement, FLCA was not a significant predictor of L2 engagement, and both components of grit, namely perseverance of effort and consistency of interest, were significant positive predictors of L2 enjoyment. In addition, FLCA was shown to be both positively and negatively connected with L2 reading comprehension, while perseverance was found to be a positive predictor of L2 reading comprehension. Between perseverance and L2 reading, interest and L2 reading, and L2 enjoyment and L2 reading, L2 engagement was revealed to have a mediating role. However, it should be noted that the L2 achievement studied in the study was L2 reading achievement, not a general achievement, therefore their findings cannot be applied to other situations like listening and reading.

Guz and Tetiurka (2016) investigated the teacher-related elements that influence positive emotions and learner engagement in FL young learners. Their findings demonstrated that a positively oriented teacher, cognitively, and emotionally engaged elicits a similar mindset in students, which might ignite engagement. Learners' engagement was also shown to be enhanced by several pedagogical practices. Learners were found to be engaged when the task they were doing was situated in a familiar environment, when the activity allowed for personalization, when the activity provided them a sense of mastery and competence, and when there was evidence of thoughtful scaffolding. The four engagement-eliciting situations substantially reflect the assumptions of the basic psychological theory (Deci \& Ryan, 2001), which states that satisfying three core psychological needs (autonomy, competence, and relatedness) can elicit engagement.

Oga-Baldwin (2019) put forward a model based on previous findings (Figure 1), which delineates the links between engagement and emotion as well as other learner-internal or -external factors. 


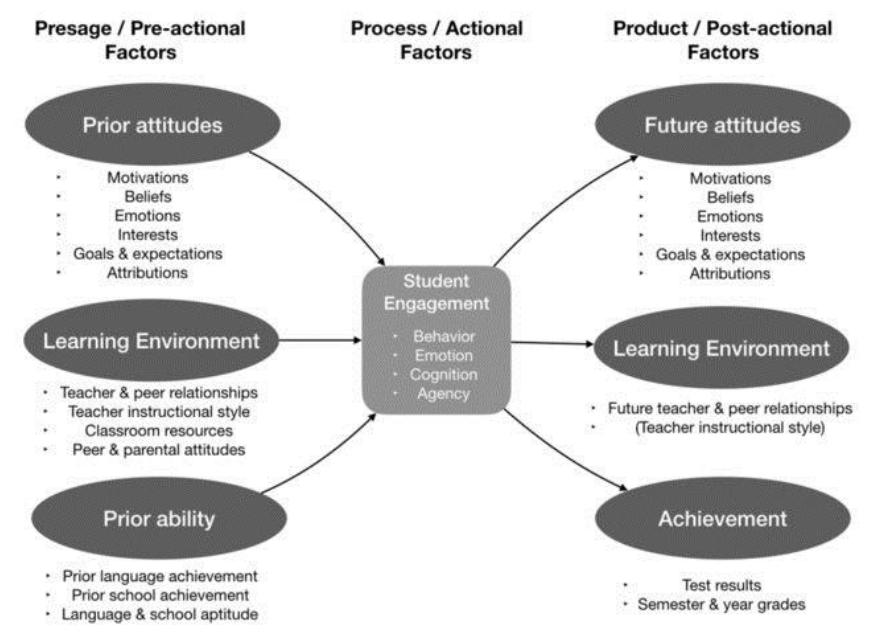

Figure 1: A contextual model of engagement (Oga-Baldwin, 2019)

Just as he argued, engagement is a result of both environmental and personal factors such as motivation and emotion and is critical in understanding achievements (Oga-Baldwin, 2019). Furthermore, since engagement indicates the absence of task-withdrawing emotions like anxiety and the presence of task-facilitating emotions like enjoyment, emotion is at the center of engagement, hence "it makes perfect sense to empirically investigate the effects of emotions on student engagement" (Dewaele \& Li, 2021).

As far as we know, no study has investigated the correlations between FLE, FLCA, and engagement in the Chinese EFL context. Previous studies on emotion, as well as engagement, are limited in several ways. First, they mainly adopted a variable-centered approach, intending to understand links between key variables as the independent and dependent variables. This method attempts to summarize patterns among participants at the group level, assuming that the finding is appliable to or representative of the greater population (Laursen \& Hoff, 2006). Using this approach makes it impossible to investigate how learners in a certain group mix emotion and engagement, in another word, the idiosyncrasy is unobservable. Such an approach may be problematic when learners do not follow the same pattern but exhibit distinct sub-patterns, and this distinctness has been evident in emotion as previously indicated (Dewaele et al., 2016; Dewaele et al., 2019). While latent profile analysis (LPA), as a person-centered approach, could reveal unobserved heterogeneous subgroups (Hagenaars \& McCutcheon, 2002). Hence makes it possible to probe into the possible combinations of positive, negative emotions and engagement in a given group.

Second, although research on achievement emotion is accumulating, and engagement is well studied in the field of education, due to the domain specificity of engagement, it is necessary to conduct research on engagement in SLA separately. It remains to be answered how emotion is related to engagement and EFL self-perceived achievement, how many possible profiles of emotion, engagement, and self-perceived achievement learners may have, for example, do learners with more positive emotion typically feel less negative emotion, have higher engagement and higher self-perceived achievement? To answer those questions, the traditional variable-based approach is somehow insufficient, hence the present study adopted a person-centered LPA to investigate the underlying profiles of Chinese EFL learners' emotion, engagement, and self-perceived achievement.

\subsection{Research Questions}

1) Are there any underlying latent profiles of FLE, FLCA, behavioral engagement, and self-perceived achievement? How many profiles can be identified?

2) How do different sub-groups differ from each other? What are the possible reasons?

\section{Methodology}

\subsection{Participants}

The data used in the current study contains 300 samples, which were retrieved randomly from a larger dataset including 633 EFL students from four Chinese senior high schools in east China, with a convenience sampling being adopted. Two are provincial key high schools, one is a province demonstrative high school, and the other is a regular high school. The four schools were chosen because they represent a range of academic levels among the participants, making the findings more representative and generalizable. In the current data, the participants are all Chinese native speakers, ranging in age from 15 to 20 , with a mean of 16 $(\mathrm{SD}=1.05)$. There are 144 males $(48 \%)$ and 156 females $(52 \%)$. Year one $(n=153,51 \%)$ and year three $(n=133$, $44.33 \%$ ) pupils make up the majority, with only 14 year two students $(4.67 \%)$.

\subsection{Instruments}

The instrument used in this study was a 5-point Likert questionnaire that included three sub-scales from previous studies: the CFLE scale (Li et al., 2018), FLCA scale (MacIntyre \& Dewaele, 2014), and the L2 behavioral engagement scale (Zhou et al., 2021); at the bottom of the questionnaire is a section for learners' self-perceived English achievements (SPA).

The CFLE scale was a modified version of the 14-item one in Dewaele \& MacIntyre (2016) by Li et al. (2018) based on Chinese samples, in which they discovered 3 dimensions, namely FLE-teacher, FLE-private, and FLE-environment. It appears to have good reliability and validity in the Chinese context.

The 8-item FLCA sub-scale in Dewaele \& MacIntyre (2014) was adopted in the present study. It was a short version of the original FLCA scale (Horwitz et al., 1986). The 8-item FLCA scale has been translated into Chinese and adopted in many studies in the Chinese EFL context (e.g., Jiang \& Dewaele, 2019), and was found to have high reliability. There are two reversed items and the scale has a single dimension.

The L 2 behavioral engagement scale used in the present study was borrowed from Zhou et al. (2020). There are 8 items with a single dimension, with three of the items reversed. In the present study, minor adjustments were made to better fit the scale in the Chinese EFL classroom (specifically, "In my 
English class..." was added to the beginning of each statement).

The final section is a 5-point Likert scale in which participants are asked to report their perceptions of their own English abilities in the classroom. 1=awful, 2=below average, 3 average, $4=$ above average, and $5=$ good. For some reasons, a self-perceived achievement rather than actual test scores were elicited in the present study: first, recent research has found a high level of consistency between self-perceived and real academic scores (Cole \& Gonyea, 2010; Kuncel et al., 2005). The self-perceived achievement was found to be the most important factor in predicting real academic performance (Hattie, 2009). Second, the participants came from four separate schools, each of which prepared and gave its own English exams, resulting in different assessments as well as standards across the four schools. As a result, actual test scores may not be comparable. Third, because the data was collected at the start of the semester when participants didn't have any exams yet, using self-perceived achievement could lessen the burden of recalling their test scores in the last semester, which is supposed to increase the response rate. Combing them together, the composite questionnaire has good composite reliability with McDonald's $\omega=0.80$.

Due to the schools' ban on cell phone use, the questionnaire was completed anonymously in a traditional paper-pen way. The demographics section of the questionnaire required information such as gender, age, and grade. The three sub-scales were then added. The SPA section is the final component. Participants were asked to rate their feelings about the objects on a scale of "1" (very unfavorable) to "5" (very favorable).

The FLE and FLCA scales were adopted with slight modifications because Chinese versions already exist ( $\mathrm{Li}$ et al., 2018; Jiang \& Dewaele, 2019). The author translated the behavioral engagement scale (Zhou et al., 2021) into Chinese, and a bilingual postgraduate student majoring in applied linguistics back-translated it into English to ensure that it was appropriate. Finally, the author discussed the final version with an applied linguistics professor.

\subsection{Procedures}

Since the data in the present study were retrieved from a larger dataset, the procedures of collecting the larger dataset will be briefly introduced. The author first got permission from all of the school's headmasters, as well as agreement from all of the participants and their parents. Each class's headteacher was responsible for distributing the questionnaires. Participants were given a brief explanation of the study's goal and were assured that their personal information would be kept private. The questionnaire was finished in 15 minutes. The headteacher then collected the questionnaires and gave them over to the author. Finally, 647 students volunteered for the study, with 14 invalid questionnaires deleted due to incomplete responses, resulting in a final sample of 633 people, and the response rate was $97.8 \%$. The present data were then retrieved randomly through SPSS 26 from the larger dataset.

\subsection{Data Analysis}

The tool for data analysis in the present study is SPSS 26, Mplus 8.3, and jamovi 2.0. Before we conducted the latent profile analysis, three separate Confirmatory Factor Analyses (CFA) were conducted by Mplus to check the construct validity of the three sub-scales in the present study. An acceptable model should have an RMSEA (Root Mean Square Error of Approximation) lower than 0.08, a CFI (Comparative Fit Index), a TLI (Tucker-Lewis Index) larger than 0.9, and an SRMR (Standardized Root Mean Square Residual) lower than 0.08 (Hu \& Bentler, 1999).

After the validation of instruments, latent profile analysis (LPA) was carried out to identify student subgroups (profiles) with homogeneous FLE, FLCA, behavioral engagement, and SPA. LPA is becoming more commonly used in numerous studies of cognitive and personal psychology and education (Bucholz et al., 2000; Keel et al., 2004; Muthén, 2001; Rasmussen et al., 2002). Since it creates internally homogeneous and externally heterogeneous subgroups using maximum likelihood estimation, this approach is an interesting alternative to other traditional analytical approaches that use a threshold on a continuous scale or some diagnostic scores (Wang et al., 2021). LPA is also a model-based approach for obtaining model fit statistics that may be used to compare models for hypothesis testing (Petersen et al., 2019). Besides the objective model fit indices, the optimal number of profiles is also based on the interpretability of results, which strengthened the reliability and validity of this method. This method assumes that 1) observations are independent of each other, making it unsuitable for hierarchical datasets with participants nested within groups; 2) covariance matrices are equal across profiles, which is similar to assumptions in linear discriminant analysis (Asparouhov \& Muth'en, 2008; Vermunt et al., 2004).

This approach has many advantages over the traditional variable-centered approach, because it could address unobserved heterogeneity within the participants, and reveal the possible patterns of emotion, engagement, and self-perceived achievement; It is a probability- and model-based approach in which profiles are categorized according to several model fit indices (e.g., BIC, AIC, entropy) using maximum likelihood estimation rather than means and standard deviations, as in the traditional method. Researchers might use these indices to assess the interpretability and representativeness of the profiles created.

LPA is suitable for the present study because previous research has indicated the independence of both FLE and FLCA, hence many possible combinations could emerge (Dewaele et al., 2016; Dewaele et al., 2019), for instance, the presence of high levels of FLE and FLCA simultaneously. Moreover, the associations between behavioral engagement, FLE, FLCA, and self-perceived achievement are underexplored, using LPA could offer insights into the typical profiles or patterns of the participants, for example, the typical engagement and FLCA profile of participants with a high FLE.

In LPA, underlying profiles or sub-groups are added stepwise, and according to the indices of fit, the researcher could judge whether the optimal solution has been obtained. The main 
data are Akaike Information Criteria (AIC), Bayesian Information Criteria (BIC), sample size adjusted BIC (aBIC), the $\mathrm{p}$-value of the Lo-Mendel-Rubin's Likelihood ratio test (LMR p), the p-value of the Bootstrap Likelihood ratio test (BLRT p), and entropy. The lower the AIC, BIC, and aBIC, the better the model fit. A significant LMR p suggests that a $\mathrm{k}$ class model is significantly better than the k-1 class model. The entropy indicates the quality of classification, and usually, a value above 0.70 is acceptable (Jung \& Wickrama, 2008). Moreover, in the present study, the appropriacy of classification will be evaluated based on the population of the smallest sub-group, which should take more than $5 \%$ of the total sample to ensure its representativeness.

\section{Results}

\subsection{Basic Statistics}

Table 1: Model fit statistics of confirmatory factor analyses (CFA)

\begin{tabular}{cccc}
\hline Fit statistics & FLE scale & FLCA scale & Engagement scale \\
\hline \hline $\boldsymbol{\chi}^{\mathbf{2}}$ & $85.4 * * *$ & $39.09 * * *$ & $32.01 * * *$ \\
\hline RMSEA & 0.06 & 0.06 & 0.07 \\
\hline CFI & 0.98 & 0.97 & 0.96 \\
\hline TLI & 0.97 & 0.96 & 0.94 \\
\hline SRMR & 0.06 & 0.03 & 0.04 \\
\hline
\end{tabular}

Note: $* * * \mathrm{p}<.001, \mathrm{RMSEA}=$ root mean-square error of approximation, $\mathrm{CFI}=$ comparative fit index, TLI=Tucker-Lewis index, SRMR=standardized root mean square.
The results of the CFA are presented in Table 1. As the table suggests, all of the sub-scales have good construct validities. The descriptives, reliability of scales as well as correlations of key variables are presented in Table 2 . The scales were all internally consistent. All of the key variables are significantly correlated, with FLE positively correlated with behavioral engagement and SPA, FLCA negatively correlated with behavioral engagement, FLE and SPA, and behavioral engagement positively correlated with SPA. The correlations are in accordance with previous findings (Dewaele \& MacIntyre, 2014, 2016; Li, et al., 2018; Jiang \& Dewaele, 2019; Su, 2019).

Table 2: Descriptive statistics (means, standard deviations, internal consistency) and correlations among the variables in this study

\begin{tabular}{c|cccc}
\hline & FLE & FLCA & Engagement & SPA \\
\hline \hline FLE & - & & & \\
\hline FLCA & $-0.39 * * *$ & - & & \\
\hline Engagement & $0.58^{* * *}$ & $-0.30^{* * *}$ & - & \\
\hline SPA & $0.47^{* * *}$ & $-0.36^{* * *}$ & $0.30 * * *$ & - \\
\hline Mean & 3.39 & 3.32 & 3.69 & 2.88 \\
\hline SD & 0.63 & 0.80 & 0.59 & 0.97 \\
\hline Cronbach's $\boldsymbol{\alpha}$ & 0.89 & 0.84 & 0.78 & \\
\hline
\end{tabular}

Note: $* * * \mathrm{P}<0.001$, SPA: self-perceived achievement

Based on the mean levels of FLE, FLCA, behavioral engagement, and SPA, the fit indices and criteria of the optimal number of clusters were shown in Table 3.

Table 3: Fit indices for different models with a varying number of latent classes.

\begin{tabular}{|c|c|c|c|c|c|c|c|}
\hline Number of profiles & AIC & BIC & aBIC & LMRp & BLRTp & Entropy & Smallest cluster freq. \\
\hline 2 & 2495.216 & 2543.365 & 2502.136 & $0.0026 * *$ & $0 * * *$ & 0.66 & $121(40 \%)$ \\
\hline 3 & 2431.487 & 2498.155 & 2441.07 & $0.0012 * *$ & $0 * * *$ & 0.74 & $45(15 \%)$ \\
\hline 4 & 2424.907 & 2510.094 & 2437.152 & 0.48 & 0.08 & 0.77 & $6(2 \%)$ \\
\hline 5 & 2417.043 & 2520.749 & 2431.95 & 0.63 & $0 * * *$ & 0.78 & $6(2 \%)$ \\
\hline 6 & 2415.454 & 2537.678 & 2433.022 & 0.14 & 0.67 & 0.75 & $15(5 \%)$ \\
\hline
\end{tabular}

Note: $* * * \mathrm{P}<0.001, * * \mathrm{p}<0.01$

As Table 3 indicates, AIC, BIC, and aBIC decreased gradually as the profile number increased, however, BIC was at the lowest level when participants were divided into 3 groups. The LMR test indicated that both 2- and 3-profile models were significantly better than 1- and 2-profile models, while 4-, 5-, and 6-profile models were not significantly better than their k-1 models. Furthermore, the smallest cluster sizes in 4-, 5-, and 6-profile models were not representative enough because they took up no more than $5 \%$ of the total participants, hence all of them were considered inappropriate. As for the 2-profile model, its entropy was lower than 0.7 , hence was screened out for its poor classification quality. Therefore, the 3 -profile model should be the optimal one in the present study.

\subsection{Profiles of FLE, FLCA, Behavioral Engagement, and Self-perceived Achievement}

Table 4: Variable means of 3-latent profile model

\begin{tabular}{c|c|c|c|}
\hline & class 1 & class 2 & class 3 \\
\hline \hline FLE & 2.676 & 4.198 & 3.498 \\
\hline FLCA & 3.726 & 2.491 & 3.359 \\
\hline Behavioral engagement & 3.162 & 4.36 & 3.745 \\
\hline Self-perceived achievement & 2.081 & 3.777 & 2.999 \\
\hline $\mathbf{n}$ & $80(26.65 \%)$ & $45(15 \%)$ & $175(58.33 \%)$ \\
\hline
\end{tabular}

Table 4 provides an overview of the 3 -profile model. As the table indicated, class 3 took up the most of the participants $(\mathrm{n}=175,58.33 \%)$, followed by class $1(\mathrm{n}=80,26.65 \%)$, and class $2(n=45,15 \%)$. According to their relative levels of FLE in the participants, class 1 was named as low-FLE profile, class 2 as high-FLE profile, and class 3 as the medium-FLE profile.

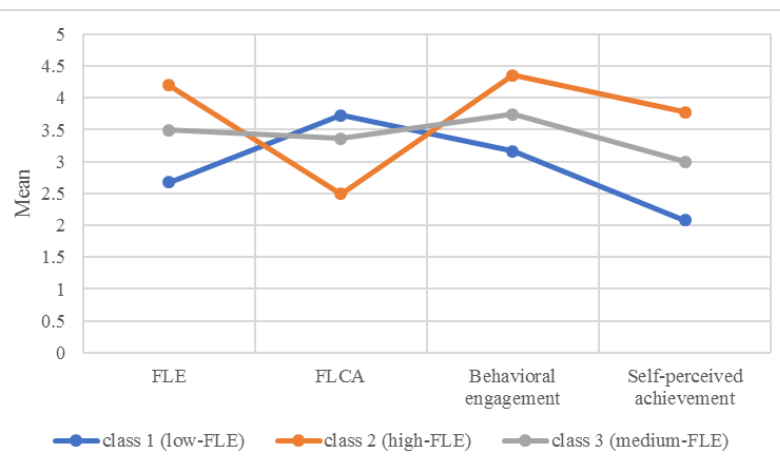

Figure 2: The three latent profiles

As visualized in Figure 2, participants in the low-FLE profile had the lowest FLE, behavioral engagement, and SPA, while they had the highest FLCA among all profiles. Participants in the high-FLE profile had the highest level of both FLE and self-perceived achievement and the lowest FLCA. Participants in the medium-FLE profile had medium levels of FLE, FLCA, behavioral engagement, and SPA.

\subsection{Inter-profile Differences}

To compare differences in the key variable among the three profiles, an ANOVA was conducted. The results of post-hoc 
pairwise comparison with Bonferroni adjustment were presented in Table 5.

Table 5: ANOVA results examining the differences across the three profiles.

\begin{tabular}{c|c|c|c}
\hline \multirow{2}{*}{ Outcomes } & Post-hoc comparison & \multicolumn{2}{|c}{ ANOVA } \\
\cline { 3 - 4 } & (Profile number) & $\mathrm{F}$ & $\eta^{2}$ \\
\hline \hline FLE & $2>3>1$ & $303.83 * * *$ & 0.67 \\
\hline FLCA & $1>3>2$ & $53.71 * * *$ & 0.27 \\
\hline Behavioral engagement & $2>3>1$ & $134.27 * * *$ & 0.48 \\
\hline SPA & $2>3>1$ & $76.02 * * *$ & 0.34 \\
\hline Note: $* * * \mathrm{P}<0.001$.
\end{tabular}

As suggested by Table 5, the differences in all the variables are significant across the three profiles. The learners in profile 2 , namely the high-FLE profile, have a significantly higher level of FLE, behavioral engagement, and SPA than those in other profiles, and the difference was also significant between the other two groups. The high-FLCA profile had a significantly higher score in FLCA than any of the other profiles, and the difference remained significant between profile 3 and 2. Interestingly, profile 3 has the medium level of all four variables. Moreover, all of the inter-profile differences appeared to have large effect sizes with an $\eta^{2}$ higher than 0.14, indicating that the differences were big. To sum up, the high-FLE profile has the highest level of FLE, engagement, and SPA; symmetrically, the low-FLE profile has the lowest level of FLE, engagement, and SPA; while the medium-FLE profile has medium levels of all the variables.

\section{Discussion}

The present study investigated the underlying profiles of Chinese high school EFL learners' FLE, FLCA, behavioral engagement, and self-perceived achievement. The present study made a contribution to the field of SLA by discovering the correlations between FLE, FLCA, behavioral engagement, and self-perceived achievement, classifying learners' differences in FLE, FLCA, behavioral engagement, and SPA with a person-centered approach, and discovering unobserved patterns of FLE, FLCA, behavioral engagement, and SPA.

The results of LPA indicated that three distinct groups could be identified: low-FLE (26.65\%), high-FLE (15\%), and medium-FLE (58.33\%). Participants in the high-FLE profile reported to have the highest level of FLE, behavioral engagement, self-perceived achievement, and lowest FLCA in all participants, in another word, they felt much enjoyment and less anxiety when learning English, and they are highly engaged in learning activities and considered themselves as having a high achievement of English. This finding dovetails with previous findings that FLE is negatively correlated with FLCA (Dewaele \& MacIntyre, 2014, 2016; Jiang \& Dewaele, 2019; Li, 2018, 2020; Li et al., 2018; Li et al., 2019), hence a higher FLE usually indicates a lower FLCA. Participants in the low-FLE profile reported low FLE, behavioral engagement, and SPA, but a high level of FLCA, which again echoes previous findings that anxious learners were less likely to engage in learning activities, less confident, and had less enjoyment. Interestingly, besides the two typical high- and low-FLE profiles, the present study found an additional medium-FLE profile. Participants in this profile reported equally medium levels in all the key variables. They felt a medium level of enjoyment, anxiety, engagement and perceived themselves as having a medium level of EFL achievement. This had not been found in previous studies, probably because scant previous research had conducted a person-centered approach like LPA. The medium-FLE profile took up most of the population $(n=175,58.33 \%)$, while the high-FLE profile took the least proportion $(n=45,15 \%)$. This may indicate that in a real Chinese EFL classroom most learners tend to feel a medium level of FLE and FLCA, and engage in the learning activities mediumly, while do not always go to the extreme and exhibit a typical pattern of high FLE and low FLCA, or low FLE and high FLCA. Additionally, the few participants in the high-FLE profile may indicate that the number of Chinese high school EFL students who felt confident in their language achievement, or found that learning English very enjoyable and getting themselves highly engaged was limited. This is probably a reflection of the general profile of Chinese EFL learners, who, when compared with international students, felt significantly less enjoyment and more anxiety (Jiang \& Dewaele, 2019; Dewaele \& MacIntyre, 2014). This may be caused by the test-oriented atmosphere in China, which is especially salient for senior high school learners. Because they are facing the very significant test of National College Entrance Examination, which could impose a tremendous impact on their future career, salary, and even social status, therefore, under a combination of social and academic pressure, learners could feel much anxious and little enjoyment, which, could, in turn, disengage them.

The present study included behavioral engagement into the nomological network of achievement emotion (Botes et al., 2021b). The results indicated that learners with a high FLE, SPA and low FLCA typically had a high level of behavioral engagement, while those with low FLE, SPA, and high FLCA had a low level of behavioral engagement. Combing the pattern with correlations between the variables, we may safely come to the conclusion that behavioral engagement is positively correlated with FLE and SPA, while negatively correlated with FLCA, a result dovetails with previous findings (Wang et al., 2016; Su, 2019). The results of ANOVA indicated that all the differences among the three profiles were significant and the effect sizes were all large, indicating that there exists a huge gap in learners' FLE, FLCA, behavioral engagement, and self-perceived achievements.

The reasons for the three latent profiles could be multifaceted and may be explained by the broaden-and-build theory (Fredrickson, 1998, 2001, 2013) and basic psychological theory (Deci \& Ryan, 1991, 2000; Ryan \& Deci, 2017). Positive emotions, according to the broaden-and-build theory, may broaden thought-cognition-action, but negative emotions can narrow it (Fredrickson, 1998). A sense of competence and relatedness, according to basic psychological needs theory, is a prerequisite of motivation and engagement (Reeve, 2012). Enjoyment has the potential to widen learners' cognitive and behavioral resources, allowing them to build greater competence and interpersonal relatedness, and therefore meet basic needs. Learners' active engagement in learning is fueled by the satisfying of basic psychological needs such as competence and interpersonal relatedness, resulting in increased behavioral engagement. Engagement is a favorable predictor of learning successes, as previous research has shown (Reschly \& Christensen, 2012; Wang et al., 2019), which explains the superior SPA of learners in the high-FLE 
profile. While FLCA, as a negative emotion, may hinder learners' resources, preventing them from meeting their basic psychological needs. As a result, it may be more difficult for learners to become involved in learning, resulting in a lower SPA, hence the low SPA and engagement in the low-FLE profile. Moreover, the influence of achievement emotions and engagement on SPA could be reciprocal. According to Oga-Baldwin (2019), affective factors such as emotions could influence learner engagement and the effect was reciprocal just as the model in Figure 1 indicated, prior emotion could influence engagement, which could intern influence the next-time emotion. The same could also be true for the other two variables, in another word, the impact of emotion on achievement, and that of engagement on achievement could all be reciprocal. The reciprocity is also evident in the control-value theory, the assumptions of which imply a reciprocal relationship between antecedents, achievement emotions, and their outcomes, including academic achievement, and all of those mutually influence each other over time (Pekrun, 2006, p.326; Pekrun et al., 2007, p.16-17). However, since the present study adopted a person-centered approach and a cross-sectional design, no causality could be assumed, and the abovementioned discussions are based on previous studies and theories.

\section{Pedagogical Implications and Limitations}

The present study could offer valuable implications for FL teachers in China. Firstly, since learners with low FLE typically have low behavioral engagement and SPA. Given the malleability of both achievement emotions and behavioral engagement (Mercer \& Dörnyei, 2020), positive psychology (Seligman, 2011) provides numerous readily teachable ways for managing emotions, and research on language learning strategies (Oxford, 1990, 2011b) has demonstrated that affective strategies are easily teachable as well. These are important techniques and approaches that may be easily shared. By instructing pupils, FL teachers may help leaners build their own emotional management skills. Secondly, it is critical for educational institutions to provide teaching and learning environments conducive to learners' engagement. Providing an emotionally secure environment in which mistakes are valued as opportunities to learn is important foundation for language learning and use (Angelovska et al., 2021). Moreover, since most of the participants in the present study appeared to only have a medium level of FLE, FLCA, engagement and SPA, it is urgent for teachers to put special attention on this population, and take actions to improve their FL learning well-being as well as engagement so as to increase their FL achievements and confidence.

Besides the implications, there are also some limitations in the present study that should be acknowledged. First, the present study is based on a limited number of students from four public senior high schools in east China, which could limit the generalizability of findings. Future studies may benefit by including more participants with diverse social-economic and educational backgrounds. Second, the present study is cross-sectional designed and person-centered approached, which makes it impossible to catch the longitudinal changes of learners' achievement emotions, engagement and achievements, and the causal relationships could also not be discovered. Future studies could make contribution by investigating whether the 3-profile pattern exists in long term, and how do they change over time. Finally, the present study used self-perceived achievement in the place of actual test scores for the comparability, which would also limit the generalizability of the findings.

\section{Conclusion}

The present study adopted the edge-cutting approach of person-centered latent profile analysis to investigate the unobserved emotion and engagement sub-groups in 300 Chinese EFL learners. The results indicated that there were 3 sub-groups within the participants, namely high-FLE profile, low-FLE profile, and medium-FLE profile. The high-FLE profile has the least population; while the medium-FLE profile took the most proportion of the participants, which indicates that special attention should be paid to this group because of its popularity. Moreover, the present study also confirms previous research by presenting the two typical patterns of low FLE-high FLCA and high FLE-low FLCA. The low-FLE profile has an especially low level of enjoyment, engagement as well as self-perceived achievement, thus more attention should also be given to this group of learners. The inter-profile differences were found to be large, which again highlights the necessity to give special attention to learners in the underperformed and anxious groups.

\section{Acknowledgement}

I want to express my sincere gratitude to all the participants in the present study as well as the reviewers of this paper.

\section{References}

[1] Alpert R., Haber R. N. (1960). Anxiety in academic achievement situations. Journal of Abnormal \& Social Psychology, 61(2): 207-215.

[2] Angelovska, T., Mercer, S. \& Talbot, K. (2021). Personality traits as predictors of language learner engagement. Language Learning in Higher Education, 11(2), 285-310.

[3] Appleton, J.J., Christenson, S.L., \& Furlong, M.J. (2008). Student engagement with school: Critical conceptual and methodological issues of the construct. Psychology in the Schools, 45, 369-386.

[4] Asparouhov, T., \& Muth'en, B. (2008). Multilevel mixture models. In G. R. Hancock, \& K. M. Samuelsen (Eds.), Advances in latent variable mixture models (pp. 27-51). Charlotte, NC: Information Age.

[5] Botes, E. (2021). Positive and negative emotions in language learning: Measurement, interactions, and nomological network. Unpublished $\mathrm{PhD}$ dissertation, University of Luxembourg.

[6] Botes, E., Dewaele, J.-M. \& Greiff, S. (2021b). Taking stock: An overview of the literature and a preliminary meta-analysis of Foreign Language Enjoyment and other individual difference variables. Unpublished paper.

[7] Botes, E., Dewaele, J.-M., \& Greiff, S. (2020a). The power to improve: Effects of multilingualism and perceived proficiency on enjoyment and anxiety in foreign language learning. European Journal of Applied Linguistics. 
[8] Bucholz, K. K., Hesselbrock, V., Heath, A., Kramer, J., \& Schuckit, M. (2000). A latent class analysis of antisocial personality disorder symptom data from a multi-centre family study of alcoholism. Addiction, 95, 553-567.

[9] Bygate, M., \& Samuda, V. (2009). Creating pressure in task pedagogy: The joint roles of field, purpose, and engagement within the interaction approaches. In Mackey, A., \& Polio, C. (Eds.). (2009). Multiple Perspectives on Interaction: Second Language Research in Honor of Susan M. Gass (1st ed.). Routledge.

[10] Christenson, S., Reschly, A. \& C. Wylie. (2012b). Preface. In S. Christenson, A. Reschly \& C. Wylie (eds.) Handbook of Research on Student Engagement. New York. NY: Springer, 2012b: v-ix.

[11] Cole, J.S., \& Gonyea, R.M. (2010). Accuracy of self-reported SAT and ACT test scores: Implications for research. Research in Higher Education, 51, 305-319.

[12] Deci, E. L., \& Ryan, R. M. (1991). A motivational approach to self: Integration in personality. In $\mathrm{R}$. Dienstbier (Ed.), Nebraska symposium on motivation: Perspectives on motivation (Vol. 38, pp. 237-288). Lincoln: University of Nebraska Press.

[13] Deci, E. L., \& Ryan, R. M. (2000). The "what" and "why" of goal pursuits: Human needs and the self-determination of behavior. Psychological Inquiry, 11, 227-268.

[14] Dewaele J-M., Dewaele L. (2017). The dynamic interactions in foreign language classroom anxiety and foreign language enjoyment of pupils aged 12 to 18 . A pseudo-longitudinal investigation. Journal of the European Second Language Association, 1(1).

[15] Dewaele J-M., Witney J., Saito K., Dewaele L. (2017). Foreign language enjoyment and anxiety: the effect of teacher and learner variables. Language Teaching Research, 22(6):676-697.

[16] Dewaele, J. \& MacIntyre, P. (2016). Foreign Language Enjoyment and Foreign Language Classroom Anxiety: The Right and Left Feet of the Language Learner. In P. MacIntyre, T. Gregersen \& S. Mercer (Ed.), Positive Psychology in SLA (pp. 215-236). Bristol, Blue Ridge Summit: Multilingual Matters.

[17] Dewaele, J. M., \& Li, C. (2020). Emotions in second language acquisition:a critical review and research agenda. Foreign Language World, (1): 16.

[18] Dewaele, J.-M. (2021) Enjoyment. In: The Routledge Handbook of Second Language Acquisition and Individual Differences. Publisher: Routledge

[19] Dewaele, J.-M., \& Alfawzan, M. (2018). Does the effect of enjoyment outweigh that of anxiety in foreign language performance? Studies in Second Language Learning and Teaching, 8(1), 21-45.

[20] Dewaele, J.-M., \& MacIntyre, P.D. (2019). The predictive power of multicultural personality traits, learner and teacher variables on Foreign Language Enjoyment and Anxiety. In M. Sato \& S. Loewen (Eds.), Evidence-based second language pedagogy: A collection of Instructed Second Language Acquisition studies (pp. 263-286). London: Routledge.

[21] Dewaele, J.-M., Botes, E., \& Greiff, S. (2020). A meta-analysis of Foreign Language Enjoyment: Taking stock after a decade of research. Unpublished paper.
[22] Dewaele, J.-M., Franco Magdalena, A., \& Saito, K (2019). The effect of perception of teacher characteristics on Spanish EFL learners' anxiety and enjoyment. The Modern Language Journal, 103, 412-427.

[23] Dewaele, J.-M., MacIntyre, P. D., Boudreau, C., \& Dewaele, L. (2016). Do girls have all the fun? Anxiety and enjoyment in the foreign language classroom. Theory and Practice of Second Language Acquisition, 2(1), 41-63.

[24] Dewaele, J.-M., Özdemir, C., Karci, D., Uysal, S., Özdemir, E. D., \& Balta, N. (2019). How distinctive is the foreign language enjoyment and foreign language classroom anxiety of Kazakh learners of Turkish? Applied Linguistics Review.

[25] Dewaele, J.-M., Witney, J., Saito, K., \& Dewaele, L. (2018). Foreign language enjoyment and anxiety in the FL classroom: The effect of teacher and learner variables. Language Teaching Research, 22(6), 676-697.

[26] Dewaele, J.-M., Witney, J., Saito, K., \& Dewaele, L. (2018). Foreign language enjoyment and anxiety: The effect of teacher and learner variables. Language Teaching Research, 22(6), 676-697.

[27] Dewaele, J.-M., \& Li, C. (2021). Teacher Enthusiasm and Students' Social-behavioral Learning Engagement: The Mediating Role of Student Enjoyment and Boredom in Chinese EFL Classes. Language Teaching Research.

[28] Dewaele, Jean-Marc \& Macintyre, Peter. (2014). The two faces of Janus? Anxiety and Enjoyment in the Foreign Language Classroom. Studies in Second Language Learning and Teaching. 4. 237-274.

[29] Dörnyei, Z., \& Kormos, J. (2000). The role of individual and social variables in oral task performance. Language Teaching Research, 4, 275-300.

[30] Finn, J.D., \& Zimmer, K.S. (2012). Student engagement: What is it? Why does it matter? In Christenson, S.L., Reschly, A.L., \& C. Wylie (Eds.), Handbook of research on student engagement (pp. 97-131). New York: Springer.

[31] Fredricks, J.A., Blumenfeld, P., \& Paris, A. (2004). School engagement: Potential of the concept, state of the evidence. Review of Educational Research, 74, 59-109.

[32] Fredrickson B L. (2001). The role of positive emotions in positive psychology: The broaden-and-build theory of positive emotions. American Psychologist, 56(3): 218-226.

[33] Fredrickson, B. L. (1998). What good are positive emotions? Review of General Psychology, 2, 300-319.

[34] Fredrickson, B. L. (2013). Updated thinking on positivity ratios. American Psychologist, 68(9), 814-822.

[35] Gkonou, C. (2017). Towards an Ecological Understanding of Language Anxiety. In C. Gkonou, M. Daubney, \& J.-M. Dewaele (Eds.), New Insights into Language Anxiety (pp. 136-156). Bristol, UK: Multilingual Matters.

[36] Guz E., Tetiurka M. (2016) Positive Emotions and Learner Engagement: Insights from an Early FL Classroom. In: Gabryś-Barker D., Gałajda D. (eds) Positive Psychology Perspectives on Foreign Language Learning and Teaching. Second Language Learning and Teaching. Springer, Cham. 
[37] Hagenaars, J. A., \& McCutcheon, A. L. (2002). Applied latent class analysis. Cambridge University Press.

[38] Hattie, J. (2009). Visible Learning: A Synthesis of Over 800 Meta-Analyses Relating to Achievement (1st ed.). Routledge.

[39] Hiver, P., Al-Hoorie, A. H., Vitta, J. P., Wu, J. (2021). Engagement in language learning: A systematic review of 20 years of research methods and definitions. Language Teaching Research.

[40] Horwitz, E.K. (2001) Language anxiety and achievement. Annual Review of Applied Linguistics, 21, 112-126.

[41] Horwitz, E.K., Horwitz, M.B., Cope, J.A. (1986) Foreign Language Classroom Anxiety. The Modern Language Journal, 70, 125-132.

[42] Hu, L., and Bentler, P. M. (1999). Cutoff criteria for fit indexes in covariance structure analysis: Conventional criteria versus new alternatives. Struct. Equ. Model. 6, $1-55$.

[43] Jiang, Y., Dewaele, J-M. (2019). How unique is the foreign language classroom enjoyment and anxiety of Chinese EFL learners? System, 82: 13-25.

[44] Jung, T., \& Wickrama, K. A. (2008). An introduction to latent class growth analysis and growth mixture modeling. Social and Personality Psychology Compass, 2(1), 302-317.

[45] Keel, P., Fichter, M., Quadflieg, N., Bulik, C., Baxter, M., Thornton, L. (2004). Application of a latent class analysis to empirically define eating disorder phenotypes. Archives of General Psychiatry, 61, 192-200.

[46] Khajavy, G. H., MacIntyre, P. D., Barabadi, E. (2018). Role of the emotions and classroom environment in Willingness to Communicate. Studies in Second Language Acquisition, 40(3), 605-624.

[47] Khajavy, Gholam Hassan. (2021). Modeling the Relations Between Foreign Language Engagement, Emotions, Grit and Reading Achievement in Student Engagement in Hiver, P., Al-Hoorie, A. H., \& Mercer, S. (Eds.). 2021:241-259. Student engagement in the language classroom. Multilingual Matters.

[48] Kuncel, N.R., Crede, M., \& Thomas, L.L. (2005). The validity of self-reported grade point averages, class ranks, and test scores: A meta-analysis and review of the literature. Review of Educational Research, 75, 63-82.

[49] Laursen, B., \& Hoff, E. (2006). Person-centered and variable-centered approaches to longitudinal data. Merrill-Palmer Quarterly, 52(3), 377-389, 1982.

[50] Li, C. (2020) A Positive Psychology perspective on Chinese EFL students' trait emotional intelligence, foreign language enjoyment and EFL learning achievement, Journal of Multilingual and Multicultural Development, 41: 3, 246-263.

[51] Li, C., Dewaele, J. \& Jiang, G. (2019). The complex relationship between classroom emotions and EFL achievement in China. Applied Linguistics Review, 11(3), 485-510.

[52] Li, C., Huang, Jian \& Li, Banban. (2020). The Predictive Effects of Classroom Environment and Trait Emotional Intelligence on Foreign Language Enjoyment and Anxiety. System.

[53] Li, C., Jiang, G., Dewaele, J.-M. (2018). Understanding Chinese high school students' Foreign Language
Enjoyment: Validation of the Chinese version of the Foreign Language Enjoyment scale. System, 76, 183-196.

[54] MacIntyre P.D. (2021) Exploring Applications of Positive Psychology in SLA. In: Budzińska K., Majchrzak O. (eds) Positive Psychology in Second and Foreign Language Education. Second Language Learning and Teaching. Springer, Cham.

[55] MacIntyre, P. D. (1995). How does anxiety affect second language learning? A reply to Sparks and Ganschow. The Modern Language Journal, 79(1), 90-99.

[56] MacIntyre, P. D., and Mercer, S. (2014). Introducing positive psychology to SLA. Studies in Second Language Learning and Teaching. 4, 153-172.

[57] MacIntyre, P., and Gregersen, T. (2012). Emotions that facilitate language learning: the positive-broadening power of the imagination. Studies in Second Language Learning and Teaching. 2, 193-213.

[58] Mercer, S. (2019) Language learner engagement: Setting the scene. In X. Gao (ed.) Second Handbook of English Language Teaching (pp. 1-19). Cham: Springer.

[59] Mercer, S., \& Dörnyei, Z. (2020). Engaging Language Learners in Contemporary Classrooms (Cambridge Professional Learning). Cambridge: Cambridge University Press.

[60] Muthén, B. (2001). Latent variable mixture modeling. In G. A. Marcoulides, \& R. E. Schumacker (Eds.). New developments and techniques in structural equation modeling (pp. 1-33). Mahwah, NJ: Erlbaum.

[61] Oga-Baldwin, W. L. Quint. (2019). Acting, thinking, feeling, making, collaborating: The engagement process in foreign language learning. System. 86. 102128.

[62] Oxford, R. L. (1990). Language learning strategies: What every teacher should know. Boston: Heinle.

[63] Oxford, R. L. (2011b). Teaching and researching language learning strategies. Harlow: Pearson Longman.

[64] Oxford, R. L. (2015). Emotion as the amplifier and the primary motive: Some theories of emotion with relevance to language learning. Studies in Second Language Learning and Teaching, 5(3), 371- 393.

[65] Pekrun R. (2006). The Control-Value Theory of Achievement Emotions: Assumptions, Corollaries, and Implications for Educational Research and Practice. Educational Psychology Review, 18(4):315-341.

[66] Pekrun, R., Frenzel, A., Goetz, T., \& Perry, R. P. (2007). The control-value theory of achievement emotions: An integrative approach to emotions in education. In P. A. Schutz \& R. Pekrun (Eds.), Emotion in education (pp. 13-36). San Diego, CA: Academic.

[67] Petersen, K. J., Qualter, P., \& Humphrey, N. (2019). The application of latent class analysis for investigating population child mental health: A systematic review. Frontiers in Psychology, 10, 1214.

[68] Philp, J., \& Duchesne, S. (2016). Exploring engagement in tasks in the language classroom. Annual Review of Applied Linguistics, 36, 50-72.

[69] Pianta, R. C., Hamre, B. K., \& Allen, J. P. (2012). Teacher-student relationships and engagement: Conceptualizing, measuring, and improving the capacity of classroom interactions. In S. L. Christenson, A. L. Reschly, \& C. Wylie (Eds.), Handbook of research on student engagement (pp. 365-386). Springer Science + Business Media. 
[70] Platt, E., \& Brooks, F. (2002). Task engagement: A turning point in foreign language development. Language Learning, 52, 365-400.

[71] Rasmussen, E. R., Neuman, R. J., Heath, A. C., Levy, F., Hay, D., \& Todd, R. D. (2002). Replication of the latent class structure of attention-deficit/hyperactivity disorder (ADHD) subtypes in a sample of Australian twins. Journal of Child Psychology and Psychiatry, 43, 1018-1028.

[72] Reeve J \& Tseng C M. (2011). Agency as a fourth aspect of students' engagement during learning activities. Contemporary Educational Psychology, 36(4): 257-267.

[73] Reeve J. (2012) A Self-determination Theory Perspective on Student Engagement. In: Christenson S., Reschly A., Wylie C. (eds) Handbook of Research on Student Engagement. Springer, Boston, MA.

[74] Ryan, R. M., \& Deci, E. L. (2017). Self-determination theory: Basic psychological needs in motivation, development, and wellness. New York, NY: The Guilford Press.

[75] Saito, K., Dewaele, J.-M., Abe, M. and In'nami, Y. (2018), Motivation, Emotion, Learning Experience, and Second Language Comprehensibility Development in Classroom Settings: A Cross-Sectional and Longitudinal Study. Language Learning, 68: 709-743.

[76] Seligman, M. (2018). PERMA and the building blocks of well-being. J. Posit. Psychol. 13, 333-335.

[77] Seligman, M. E. P. (2011). Flourish: A visionary new understanding of happiness and well-being. New York: Atria/Simon \& Schuster.

[78] Shernoff, D.J. (2013). Optimal learning environments to promote student engagement. New York: Springer.

[79] Sinatra, G. M., Heddy, B. C., \& Lombardi, D. (2015). The challenges of defining and measuring student engagement in science. Educational Psychologist, 50(1), $1-13$

[80] Skinner E. A., Kindermann T. A., Furrer C. J. (2009). A Motivational Perspective on Engagement and Disaffection Conceptualization and Assessment of Children's Behavioral and Emotional Participation in Academic Activities in the Classroom. Educational \& Psychological Measurement, 69(3): 493-525.

[81] Skinner, E.A., \& Pitzer, J.R. (2012). Developmental dynamics of student engagement, coping, and everyday resilience. In Christenson, S.L., Reschly, A.L., \& C. Wylie (Eds.), Handbook of research on student engagement (pp. 21-44). New York: Springer.

[82] Spielberger, C.D. and Reheiser, E.C. (2009), Assessment of Emotions: Anxiety, Anger, Depression, and Curiosity. Applied Psychology: Health and Well-Being, 1: 271-302.

[83] Su, Qi. (2019). An SEM Study of College Students' Engagement in English Learning. Foreign Language Teaching, (01), 83-88+47.

[84] Svalberg, A.M.-L. (2009). Engagement with language: Interrogating a construct. Language Awareness, 18(3-4), 242-258.

[85] Vermunt, J. K., Lewis-Beck, M., Bryman, A., \& Liao, T. (2004). Latent profile model. The Sage Encyclopedia of Social Sciences Research Methods, 554-555.

[86] Wang, M. T., Fredricks, J. A., Ye, F., Hofkens, T. L., \& Linn, J. S. (2016). The math and science engagement scales: scale development, validation, and psychometric properties. Learning \& Instruction, 16-26.

[87] Wang, Y., Chung, M. C., \& Wang, N. (2021). Parenting revisited: Profiles and associations with psychological distress among traumatized Chinese adolescents. Current Psychology, 1-10.

[88] Zhou, Ashlee Shiyao \& Hiver, Philip \& Al-Hoorie, Ali. (2021). Measuring L2 Engagement: A Review of Issues and Applications. In Hiver, P., Al-Hoorie, A.H., \& Mercer, S. (Eds.) (2021b). Student engagement in the language classroom (p.75-98). Clevedon: Multilingual Matters. 